\title{
La modélisation thermique de transistor a haute puissance de type HEMT
}

\section{Thermal modeling of high power transistor HEMT type}

\author{
Abdelhamid Amar ${ }^{1,2}$, Bouchaïb Radi ${ }^{2}$, Abdelkhalak El Hami ${ }^{3}$ \\ ${ }^{1}$ Laboratoire de Mécanique de Normandie LMN, INSA Rouen, France, Laboratoire d'Ingénierie, Management \\ Industriel et Innovation (IM2I) FSTS, Maroc amarabdelhamid20@gmail.com \\ ${ }^{2}$ Laboratoire d'Ingénierie, Management Industriel et Innovation (IM2I) FSTS, Maroc, bouchaib.radi@yahoo.fr \\ ${ }^{3}$ Laboratoire de Mécanique de Normandie LMN, INSA Rouen, France, abdelkhalak.elhami@insa-rouen.fr
}

RÉSUMÉ. La mécatronique est une discipline qui combine entre la mécanique, l'électronique et l'informatique. L'apparition des systèmes mécatronique donne naissance à des phénomènes de défaillance et de dégradation qui se développe avec le temps et qui ne sont pas bien maitrisées. Pour étudier ces défaillances on va utiliser la méthode des éléments finis, est un schéma (méthode) numérique qui permet de simuler (résoudre) via l'outil informatique des problèmes de la physique compliqués. Et ce, en approximant le modèle mathématique basé sur une équation aux dérivées partielles dont le nombre d'inconnus est infini par un modèle algébrique matriciel dont le nombre d'inconnus est fini. La mise en application de cette méthode ce fait avec deux logiciels COMSOL et ANSYS, et les simulations vont nous permettre d'observer le comportement de notre composant ainsi détecter l'origine des défaillances.

ABSTRACT. Mechatronics is a discipline that combines mechanics, electronics and computer science. The appearance of mechatronic systems gives rise to failure and degradation phenomena that develop over time and are not well controlled. To study these failures we will use the finite element method, is a numerical scheme (method) that allows to simulate (solve) complicated physics problems via the computer tool. This is made by approximating the mathematical model based on a partial differential equation whose number of unknowns is infinite with a matrix algebraic model whose number of unknowns is finite. The implementation of this method is made with two software programs COMSOL and ANSYS, and the simulations will allow us to observe the behavior of our component and detect the origin of the failures.

MOTS-CLÉS. Modélisation thermique, système mécatronique, transistor HEMT, performance, température, puissance dissipée.

KEYWORDS. Thermal modelling, mechatronic systems, transistor HEMT, performance, temperature, power dissipated.

\section{Introduction}

La conception robuste des systèmes mécatronique est un enjeu technique qui est très important, dont il est nécessaire de prendre en considération les défaillances qui appariaient lors des premières versions de fabrication avant la fabrication des nouvelles versions, dans ce cadre la simulation numérique ne permet d'observer et avoir beaucoup d'information sur ces défaillances, en utilisant la méthode des éléments finis, avec des logiciels comme ANSYS et COMSOL. Avec tous ces outils on peut détecter les défaillances d'un système à développer dès la période de la conception avant d'arriver à l'étape de la fabrication, on subit notre système à toutes les conditions d'emploi et de l'environnement. Parmi les composants très importants dans ces systèmes, on trouve le transistor. Il est un élément électronique qui a créé une révolution dans la conception et le développement de tels systèmes en assurant la commande, l'amplification et l'abaissement des signaux. Quand on parle des systèmes les plus développés comme les systèmes aéroportés, on constate que la plupart de ses défaillances vient de ses amplificateurs à haute puissance (HPA).

D'après différents travaux, un grand pourcentage de ces défaillances est dû à des transistors à haute puissance, on cite l'enterrement de grille, dégradation des contacts ohmiques, déformation de la couche $\mathrm{AlGaN}$... ces défaillances ont une relation avec la température de fonctionnement qui 
dépasse les valeurs critiques par l'échauffement des transistors. Donc la température de fonctionnement a une grande influence sur la performance de ces derniers. Dans ce travail, on va proposer un modèle thermique du transistor bien réaliste dans des conditions similaires à celles de l'environnement de fonctionnement, puis on va utiliser la méthode des éléments finis avec le logiciel COMSOL pour faire de la simulation numérique afin d'observer la distribution de la température sur toute la structure. Dans la suite on va entamer une étude de comportement thermomécanique grâce à la combinaison entre l'étude thermique et mécanique.

\section{Les systèmes mécatroniques.}

L'apparition des systèmes mécatroniques est une révolution pour le monde industriel, il affecte de plus en plus le monde du transport et en particulier le secteur automobile. L'utilisation de ces systèmes se généralise rapidement et influence maintenant tous les secteurs de l'industrie. La mécatronique a bouleversé la conception et la fabrication des systèmes complexes. En particulier, son introduction dans le secteur automobile a profondément modifié les processus de développement et de fabrication. Ainsi, la voiture n'est plus conçue commeun dispositif mécanique qui porte quelques commandes électroniques, mais comme un système mécatronique, où les composants des différentes technologies sont entièrement intégrés [ALI 10].

\section{Les transistors à haute puissance}

Parmi les composants les plus importants dans un système mécatronique, on trouve les transistors, et lorsqu'il s'agit d'un système aéronautique ou aéroporté, on parle donc des transistors à haute puissance, dont on trouve plusieurs types. Le transistor J-FET, Le transistor MESFET, MOSFET, Le Transistor à Haute Mobilité Électronique (HEMT) [GWE 10] [1].

\subsection{Le Transistor à Haute Mobilité Électronique (HEMT).}

Le transistor HEMT apparait comme une évolution majeure du MESFET. La différence est que le HEMT utilise une hétérojonction, c'est à dire une jonction entre des matériaux ayant des bandes d'énergie différentes, de manière à faire passer les électrons constituant le courant drain-source dans un semi-conducteur non-dopé, afin de diminuer le temps de transit et donc augmenter les performances en fréquence. La vitesse des électrons est en effet d'autant plus grande que le dopage du semi-conducteur est faible, car la dispersion d'impuretés ionisées est réduite [SAN 05] [2].

\subsection{La topologie de HEMT}

D'après la recherche faite dans ce cadre, on a trouvé que le transistor HEMT possède plusieurs topologies comme le montre la figure suivante.

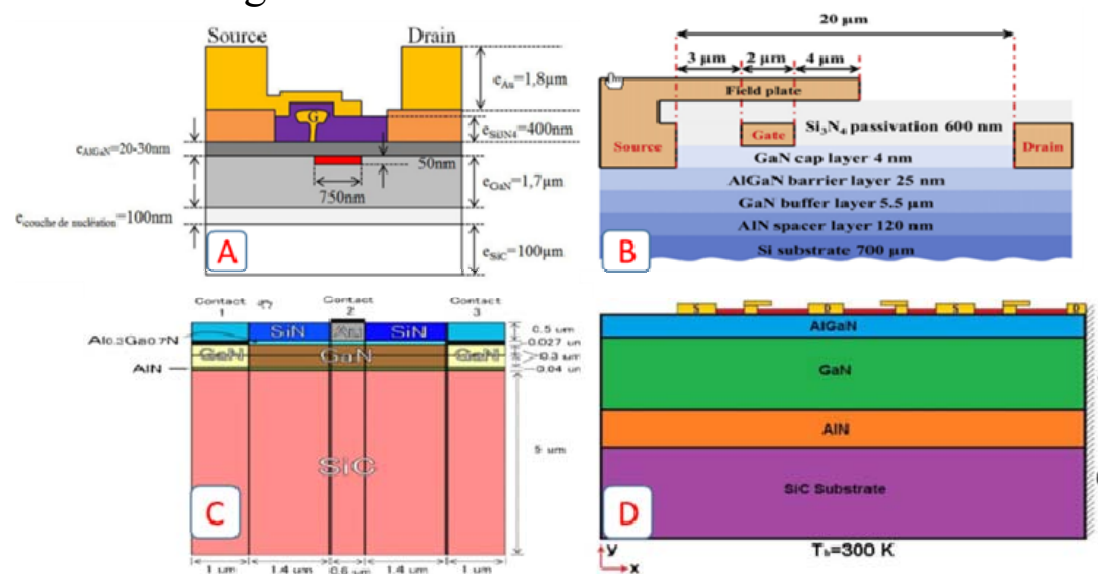

Figure 1. Différentes topologie de transistor HEMT 
La structure (A) a été utilisé dans la Thèse de Lény Baczkowski[LEN 10], pour faire une modélisation thermique de ce type de transistor. Stone Cheng a considerer la structure (B) dans son article nomé " Novel packaging design for high-power GaN-on-Si high electron mobility transistors (HEMTs) "[STO 13], pour étudier Les effets de la conception de la structure et de son procédé de fabrication sur les performances du dispositif et de l'appareil, Sa résistance thermique a été étudiée aussi .L'auto-échauffement dans les structures de dispositifs AlGaN/GaN a été de mesurée par thermographie infrarouge (IR) et spectroscopie micro-Raman. Dans un travail sous-titre " modelisation numerique des effets thermiques dans le transistor hemt en thechnologie gan » [FRA 13] FARADJI et SENOUCI ont choisi la structure (C) pour faire une étude thermique sur ce transistor en utilisant une méthode numérique à l'aide de logiciel SILVACO. La structure (D) a été utilisée pour faire la modélisation thermique par éléments finis de dispositifs avec différentes tailles de plaques de champ, les données de distribution de chaleur obtenues à partir de données de simulations en 2D, dans un article nommé « effects of field plate on the maximum temperature and temperature distribution for gan hemt devices » de Dogacan Kara [KAR 16].

\subsection{Les différentes couches de HEMT}

Le transistor HEMT est composé de plusieurs couches des différents matériaux, comme le montre la figure suivante

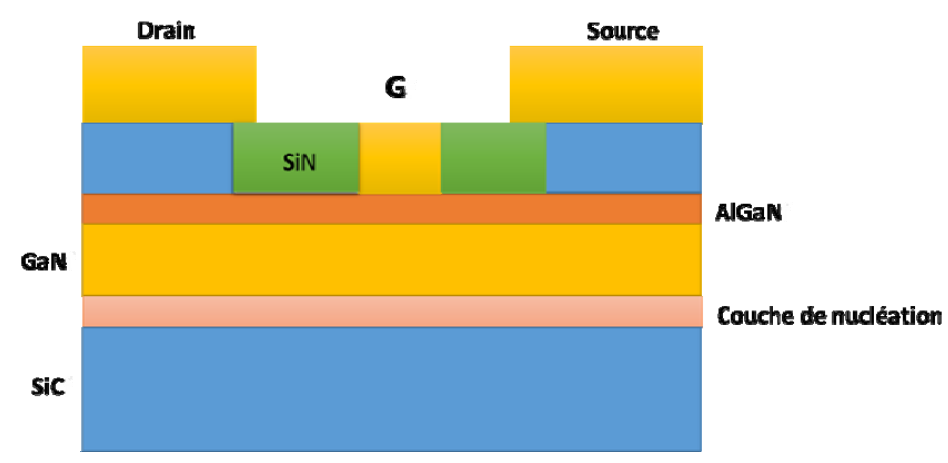

Figure 2. Différentes couches de transistor HEMT

Chacune de ces couche de matériaux assure une fonction bien déterminée :

- - la couche SIC est utilisée afin de constituer le substrat, est un support en silicium sur celui-ci que sera réalisé le composant.

- - la couche interface : placée entre le substrat et la couche de $\mathrm{GaN}$ sera requise de manière à passer graduellement de l'accord de maille du GaN vers celui du Si

-- la couche GaN assure : La couche de nitrure de gallium est placée juste au-dessous de la couche ternaire. Elle contient le 2DEG d'électrons dans sa partie supérieure, répartis sur une épaisseur de quelques nanomètres

- - la couche AlGaN : une barrière qui fait quelques dizaines de nanomètres. La différence de gaps entre ce composé ternaire et le matériau en Nitrure de Gallium crée une hétérojonction dans laquelle les électrons seront confinés afin de constituer le gaz bidimensionnel d'électrons plus connu sous le nom de 2DEG [FRA 13].

\subsection{Principe de fonctionnement de HEMT}


L'idée de base à l'origine de ce composant est d'utiliser comme canal conducteur d'un transistor à effet de champ, un gaz bidimensionnel (gaz-2D) d'électrons circulant dans un matériau peu dopé et résultant de l'occupation des niveaux d'énergie du puits de potentiel caractéristique d'une hétérojonction. Trois électrodes communément dénommés : source, grille et drain permettant de contrôler le courant et la tension de fonctionnement de transistor. Les contacts de sources et de drain sont de type ohmique, le contact de grille et de type Schottky. Pour les applications concernant l'amplification de puissance le montage source commune est utilisé, l'électrode de source étant connecté à la masse. La grille permet de contrôler la densité de courant circulant dans le transistor en agissant électro-statiquement sur le gaz d'électrons situé à son aplomb : c'est l'électrode de commande basse puissance. Le drain permet de contrôler la tension de fonctionnement du composant : c'est l'électrode de commande forte puissance [LEN 10].

\section{La modélisation thermique de HEMT}

Dans cette partie on va étudier le comportement thermique dans le transistor HEMT, en premier temps on va faire un petit rappel de quelques notion fondamentale dans la thermique tels que les modes de transfert thermiques et autres, et par suite on va faire des simulations thermiques avec deux logiciel ANSYS et COMSOL et on finira par une discussion des résultats obtenus.

\subsection{Le transfert thermique}

L'étude des transferts thermiques permet de quantifier les échanges énergétiques entre un système et un autre, en déterminant les valeurs caractéristiques des variables d'état du système et souvent la température. Pour réaliser cette étude on doit savoir les différents termes dans domaine thermique :

- La chaleur : un échange d'énergie qui se traduit par la propagation d'énergie au sein d'un corps par l'agitation désordonnée des atomes.

- Le champ de température : Les transferts d'énergie sont déterminés à partir de l'évolution dans l'espace et dans le temps de la température : La valeur instantanée de la température en tout point de l'espace est un scal-aire appelé champ de température.

$$
T=f(x, y, z, t) .
$$

Nous distinguerons deux cas si le champ de température indépendant du temps donc le régime est dit permanent ou stationnaire. Si l'évolution du champ de température avec le temps donc le régime est dit variable ou transitoire

Le gradient de température : Si l'on réunit tous les points de l'espace qui ont la même température, on obtient une surface dite surface isotherme. La variation de température par unité de longueur est maximale le long de la normale à la surface isotherme. Cette variation est caractérisée par le gradient de température.

- Flux et densité de flux de chaleur : On appelle flux de chaleur, la quantité de chaleur transmise sur la surface $\mathrm{S}$ par unité de temps :

$$
\phi=\frac{d Q}{d t}
$$

\subsection{Les modes de transfert de chaleur}

Le flux de chaleur peut être transféré par conduction, par convection et/ou par rayonnement : 
- La conduction : Le transfert d'énergie par conduction à travers une surface A [m2] est caractéristique des transferts thermiques entre solides ou au sein d'un solide .La loi de Fourier relie le flux de chaleur et le gradient de température :

$$
\phi=\lambda . S \cdot \frac{d T}{d x}
$$

- La convection : C'est le transfert de chaleur entre un solide et un fluide, l'énergie étant transmise par déplacement du fluide. Ce mécanisme de transfert est régi par la loi de Newton :

$$
\phi=S \cdot h\left(T_{p}-T_{\infty}\right)
$$

Avec $T_{s}:$ La température à la surface.

$T_{\infty}$ : La température à une distance suffisamment grande du système pour que la température soit Indépendante de la température du système [JAN 16].

- Le rayonnement : C'est un transfert d'énergie électromagnétique entre deux surfaces (même dans le vide). Dans les problèmes de conduction, on prend en compte le rayonnement entre un solide et le milieu environnant et dans ce cas nous avons la relation :

$$
\phi=\varepsilon \cdot \sigma \cdot S\left(T_{A}^{4}-T_{\infty}^{4}\right)
$$

Dans ce cas, le transfert de chaleurs par la convection et le rayonnement ne représentent que $1 \%$, alors que le reste se fait par conduction le mode dominant, dans la suite d'étude on va se baser sur l'observation de transfert de chaleur par conduction au niveau de la structure [SAN 05].

\subsection{Simulation thermique du transistor HEMT}

Dans cette partie on va traiter les étapes de la simulation thermique qu'on a réalisé avec les deux logiciels ANSYS et COMSOL, et la structure choisie ainsi les propriétés des différentes couches.

\subsubsection{Les propriétés physiques des matériaux}

Les matériaux utilisés dans la construction de notre structure, possèdent des propriétés physiques [JAR 14], qui sont présentés dans ce tableau suivant :

\begin{tabular}{|c|c|c|c|}
\hline Matériaux & $\begin{array}{c}\text { Densité volumique } \boldsymbol{\rho} \\
{[\mathbf{k g} / \mathbf{m} 3]}\end{array}$ & $\begin{array}{c}\text { Conductivité thermique } \\
\text { KTH }[\mathbf{W} / \mathbf{m} / \mathbf{K}]\end{array}$ & $\begin{array}{c}\text { Capacité thermique } \\
\text { massique cp [J/kg/K] }\end{array}$ \\
\hline Au & 19300 & 310 & 137 \\
\hline SiN & 3300 & 10 & 713 \\
\hline AlGaN & 5470 & $25 \cdot\left(\frac{293}{273+T}\right)^{-1,35}$ & 548 \\
\hline GaN & 6100 & $161 \cdot\left(\frac{293}{273+T}\right)^{-1,45}$ & 490 \\
\hline SiC & 3220 & $416 \cdot\left(\frac{293}{273+T}\right)^{-1,5}$ & 690 \\
\hline Interface GaN/SiC & 6100 & $6,7 \cdot\left(\frac{293}{273+T}\right)^{-2,7}$ & 490 \\
\hline
\end{tabular}




\subsubsection{Conditions aux limites}

Pour assurer la mise en place de la méthode des éléments finis, on doit définir les conditions aux limites qui seront imposé à notre transistor, elles nous permettent de simuler le système en présence des conditions de fonctionnement, on a deux conditions importantes :

- La température de référence : cette température est variable selon l'environnement ou se situe notre système ; dans notre cas on va prendre $300 \mathrm{~K}$ comme température de référence.

- La puissance dissipée : cette condition représente la charge appliquée sur notre système, elle est sous forme de chaleur, elle est imposée au niveau de la zone active de transistor, qui se situe à la sortie de grille à côté de drain.

\subsubsection{Influence de la puissance dissipée.}

Pour étudier l'influence de la puissance dissipée sur la variation de température de composant on va varier la valeur de la puissance dissipée, au niveau de la zone active de 1 à $9 \mathrm{~W}$, on fixe la température de référence en $25^{\circ} \mathrm{C}$ et on observe la variation de température au niveau de composant en tirant la valeur maximale à chaque fois.

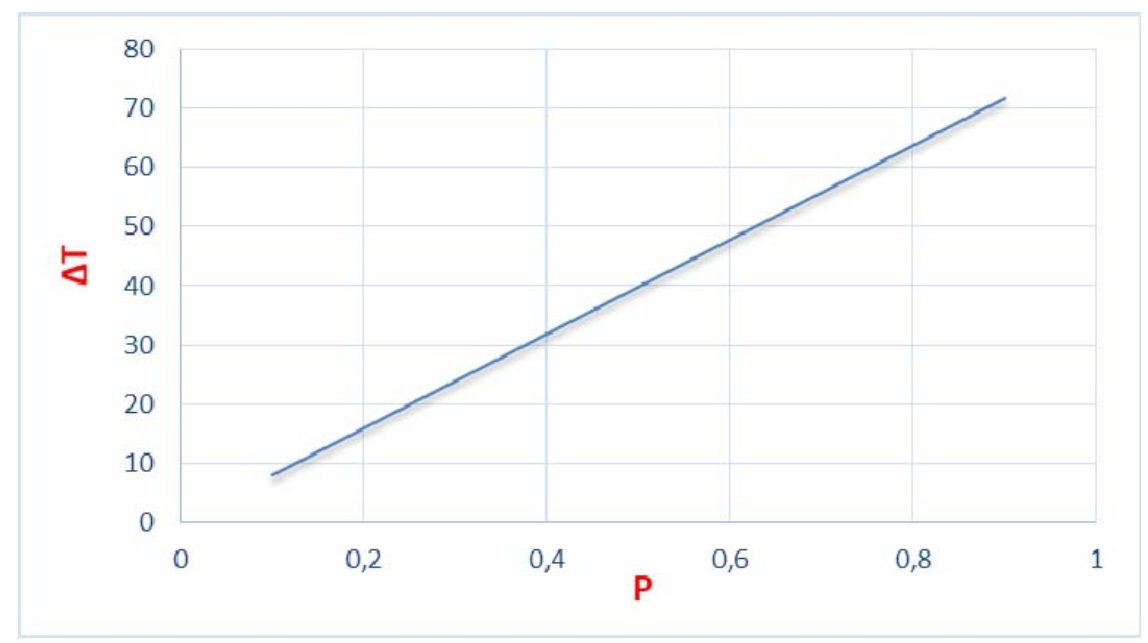

Figure 2. Variation de la température de transistor HEMT

D'après la figure, on remarque que la température maximale augmente en fonction de la puissance dissipée. ce qui signifie que cette puissance a une influence très importante sur la température de fonctionnement qu'il faut prendre en considération, car ça aura un impact sur le fonctionnement et la performance de transistor, dans le cas où la température maximale dépasse une valeur critique, il ramène a des défaillances d'origine thermique, comme l'enterrement de grille à cause de la diffusion de son métal, et la dégradation des contacts Schottky, et l'electromegration au niveau des contacts ohmique [MEN 08]. Et autres defaillance qui sont al'etude jausqu'a aujourdhui .

La maitraise de ces dfaillances permet d'augmenter la fiabilité de ces transistor donc la fiabilité et performance et d'assurer une fabricaion robuste des système mecatronique.

\section{Conclusion}

Dans cet article on a fait une modelisation thermique du transistor a haute puissance qui est l'un des composantes essentiels dans un système mecatronique. Elle a permis d'observer l'influence de la puissance dissipée sur la temperature maximale de fonctionement de transistor.en utilisant le modele élements finis resolus par le logicile COMSOL. 
Afin d'assurer une fabrication robuste des transistors a haute puissance donc celle des système mecatronique ,il faut faire une etude d'ptimisation de la puissance dissipée, il faut etudier autres types de defaillances d'autres origines.

\section{Bibliographie}

[ALI 10] A. G. Mihalache « Modélisation et évaluation de la fiabilité des systèmes mécatroniques : application sur système embarqué », 2010.

[GWE 10] G. Le Coustre., «Contribution au développement d'une filière de transistor de forte puissance à base de technologie HEMT GaN pour applications télécoms et radar » Lille, 2010.

[SAN 05] Sandra DE MEYER., «Etude d'une nouvelle filière de composants HEMTs sur technologie nitrure de gallium. » UNIVERSITE DE LIMOGES, 2005

[LEN 10] Lény BACZKOWSK., "Modélisation et Caractérisation Thermique de Transistors de Puissance Hyperfréquence GaN et Conséquences sur la Fiabilité de Modules Radars d'Émission/Réception » Université des sciences et Technologies de Lille, 2015

[STO 13] S. Cheng and P. C. Chou, "Novel packaging design for high-power GaN-on-Si high electron mobility transistors (HEMTs)," Int. J. Therm. Sci., vol. 66, pp. 63-70, Apr. 2013.

[FRA 13] FRADJI and SENOUCI, «modélisation numérique des effets thermiques des effets thermiques dans le transistor HEMT en technologie gan», 2013

[KAR 16] KARA.D, N. Donmezer, T. F. Canan, O. Sen, and E. Ozbay, "Effects of Field Plate on the Maximum Temperature and Temperature Distribution for GaN HEMT Devices," 2016.

[JAN 16] JANNOT.Y and C. Moyne, “Transferts thermiques,” EdiLivre, p. 298, 2016.

[JAR 14] Jardel.O et al., "InAlN/GaN HEMTs based L-band high-power packaged amplifiers," Int. J. Microw. Wirel. Technol., vol. 6, no. 6, pp. 565-572, Dec. 2014.

[MEN 08] G. Meneghesso et al., "Reliability of GaN high-electron-mobility transistors: State of the art and perspectives," IEEE Trans. Device Mater. Reliab., vol. 8, no. 2, pp. 332-343, Jun. 2008. 